\title{
Natural Product as a Source of Lead to the Design of New Drugs \\ Harshal Ashok Pawar*
}

Assistant Professor and HOD (Quality Assurance), Dr. L. H. Hiranandani College of Pharmacy, Ulhasnagar-421003, Maharashtra, India

\begin{abstract}
Natural products have been a source of medicinal agents since thousands of year and remarkable numbers of modern drugs have been derived from natural sources, predominantly based on their knowledge available in traditional medicine. The modern natural product research is undergoing a revolution due to recent advancements in combinatorial biosynthesis, microbial genomics and screening processes. The total synthesis of complex natural products has long presented a big challenge to the synthetic chemist, and led to considerable advances in synthetic methodology. To continue to be competitive with other drug discovery methods, natural product research needs to continually improve the speed of the screening, isolation, and structure elucidation processes, as well addressing the suitability of screens for natural product extracts and dealing with issues involved with large-scale compound supply.
\end{abstract}

Keywords: Natural products; Quinine; Artemisia annua; Camptothecin; Taxol

\section{Introduction}

Natural products have been a source of medicinal agents since thousands of year and remarkable numbers of modern drugs have been derived from natural sources, predominantly based on their knowledge available in traditional medicine. The past century, however, has seen an increasing role played by microorganisms in the production of the antibiotics and other drugs for the treatment of serious diseases, and more recently, marine organisms have proved to be a rich source of novel bioactive agents. Natural products will continue to play a crucial role in meeting this demand through the expanded investigation of the world's biodiversity, much of which remains unexplored.

It has been projected by the World Health Organization (WHO) that approximately $80 \%$ of the world's population relies mainly on traditional medicines for their primary health care. Plant products also play an imperative role in the health care systems of the remaining $20 \%$ of the population, mainly residing in developed countries, and at least 119 chemical substances, derived from 90 plant species, can be considered as important drugs at present in use in one or more countries. Of these 119 drugs, $74 \%$ were discovered as a result of chemical studies aimed at the isolation of the active substances from plants used in traditional medicine [1].

The debt owed by combinatorial chemistry to organic synthesis is obvious and widely recognized by practitioners of either field. As combinatorial chemistry matures, it is becoming clear that combinatorial chemistry is also fuelling major improvements in organic synthesis. The scope and limitations of a number of venerable reactions is now much better understood with their incorporation into combinatorial sequences for the generation of compound libraries. Many of the techniques developed by combinatorial chemists for parallel synthesis and purification can be profitably employed even when making one compound at a time. Combinatorial chemistry will also have a growing impact on natural product total synthesis, an area that has primarily focused on a target molecule as an end in itself rather than an exciting avenue for discovering new compounds with new properties.

Novel natural product derivatives can be optimized on the basis of their biological activities using modern sophisticated biosynthetic, combinatorial and high throughput techniques to yield effective chemotherapeutic and other bioactive agents. The phytochemists have always been impressed by the fact that compounds found in nature exhibit an almost incredible range of diversity in terms of their physicochemical and biological properties. Most of these compounds are secondary metabolites with functions in plants, fungi, and marine organisms that are still not extensively understood. At present, it is assumed that many of these compounds act in defense of the harmful effects of toxins, carcinogens, or mutagens found in the plant or attack by external predators [2-4].

The modern natural product research is undergoing a revolution due to recent advancements in combinatorial biosynthesis, microbial genomics and screening processes. Moreover, access to hyphenated techniques like Liquid Chromatography-Mass Spectrometry, Liquid Chromatography-Nuclear Magnetic Resonance have raised the hope of drastically reducing the time and cost involved in natural product research by using dereplication processes that are combination of techniques to avoid the already reported compounds. The developments of antimalarial and anticancer drugs using some of the lead compounds (Phytoconstituent) isolated from natural source are discussed below.

\section{Development of Antimalarial Drugs Using Natural Lead}

Quinoline type of alkaloid, quinine, formed the basis for the synthesis of the commonly used antimalarial drugs, chloroquine and mefloquine. Chemically quinine (Figure 1) is (R)-(6-Methoxyquinolin4-yl) ((2S,4S,8R)-8-vinylquinuclidin-2-yl)methanol. It was originally isolated in 1820 by French pharmacists Caventou and Pelletier from the bark of Cinchona species (e.g., Cinchona officinalis) which had long been used by native groups in the Amazon region for the treatment of fevers, and was first introduced into Europe in the early 1600s for the treatment of malaria.

With the emergence of resistance to these drugs in many tropical regions, another plant, Artemisia annua (Quinhaosu), long used in the treatment of fevers in traditional Chinese medicine, has yielded the agents artemisinin. Chemically artemisinin (Figure 2) [(3R,5aS,6R,8aS,9R,12S,12aR)-octahydro-3,6,9-trimethyl-3,12-epoxy-

*Corresponding author: Harshal Ashok Pawar, Head of Department (Quality Assurance), Dr. L. H. Hiranandani College of Pharmacy, Smt. CHM Campus, Opp. Ulhasnagar Railway Station, Ulhasnagar-421003, Maharashtra, India, Tel: +91-8097148638; E-mail: harshal.dlhhcop@gmail.com

Received September 09, 2014; Accepted October 24, 2014; Published October 26, 2014

Citation: Pawar HA (2014) Natural Product as a Source of Lead to the Design of New Drugs. Nat Prod Chem Res 2: 156. doi:10.4172/2329-6836.1000156

Copyright: (c) 2014 Pawar HA. This is an open-access article distributed under the terms of the Creative Commons Attribution License, which permits unrestricted use, distribution, and reproduction in any medium, provided the original author and source are credited. 
<smiles>C=CC1CC2CCC(C1)N2CC[C@H](O)c1ccnc2ccc(OC)cc12</smiles>

Figure 1: Chemical structure of Quinine<smiles>C[C@H]1CC[C@H]2[C@@H](C)C(=O)O[C@@H]3O[C@]4(C)CC[C@@H]1[C@]32OO4</smiles>

Figure 2: Chemical structure of Artemisinin.

12H-pyrano[4,3-j]-1,2- benzodioxepin-10(3H)-one]. Artemisinin and its derivatives, artemether and arteether, are exclusively important antimalarial agents valuable for treating otherwise resistant parasites $[5,6]$.

They act more rapidly and over a wider range of parasite developmental stages than other antimalarials [7]. In recent times, a study has shown that artesunate reduces mortality compared with quinine in the treatment of severe Plasmodium falciparum malaria [8], even though this study has raised some interesting issues $[9,10]$. Like quinine, artemisinin is also derived from a plant but is structurally a most dissimilar compound. The pharmacophore consists of a peroxide within a 1,2, 4-trioxane configuration, the presence of which has led to several suggestions of how these antimalarials might work [11]. Many of these suggestions have been based on the understanding of classic peroxide chemistry, and much of the experimental evidence that has been accumulated is based on the chemical behavior of artemisinins $[12,13]$. However, the application of medicinal chemistry principles rather than any prior consideration on how the peroxide exerts its antimalarial activity has led to improved forms of artemisinins (both in terms of potency and toxicity) and to the successful synthesis of artemisone [14-17], which is one of the most potent semi-synthetic derivates that is undergoing clinical studies. Experimental chemistry has also generated fully synthetic trioxolanes, which are currently being developed for clinical use [18]. None of these approaches has required knowledge of the mechanisms of action of these drugs. Poor bioavailability of artemisinin limits its effectiveness and hence semisynthetic derivatives of artemisinin have been developed. These include:

- Artesunate (water-soluble: for oral, rectal, intramuscular, or intravenous use)

- Artemether (lipid-soluble: for oral, rectal or intramuscular use)

- Dihydroartemisinin

- Artelinic acid

- Artemotil

\section{Development of Anticancer Drugs Using Lead from Natural Source}

Camptothecin $\quad\left[(\mathrm{S})\right.$-4-ethyl-4-hydroxy-1H-pyrano $\quad\left[3^{\prime}, 4^{\prime}: 6,7\right]$ indolizino [1,2-b]quinoline-3,14- $(4 \mathrm{H}, 12 \mathrm{H})$-dione $]$ is secondary metabolites isolated from the wood bark of Camptotheca acuminata. This drug is native of China. Taxol is chemically $(2 \alpha, 4 \alpha, 5 \beta, 7 \beta, 10 \beta, 13 \alpha)-4,10$ bis(acetyloxy)-13-\{[(2R,3S)-3-(benzoylamino)-2-hydroxy-3phenylpropanoyl]oxy\}-1,7- dihydroxy-9-oxo-5,20-epoxytax-11-en2 -yl benzoate. This drug was obtained from the bark of plant species, Taxus brevifolia (Pacific Yew), found in the northwest Pacific coastal region of the United States. The compounds were isolated guided by bioassay on various extracts and chromatographic fractions. Their unique and hitherto known structures (Figures 3 and 4 ) were elucidated by nuclear magnetic resonance, mass spectrometry, and X-ray analysis.

Both compounds have exceptional mechanisms of antitumor activity; camptothecin uniquely inhibits an enzyme, topoisomerase I, involved in DNA replication. Taxol binds to a protein, tubulin, thus inhibiting cell division. Taxol has been called the best new anticancer agent developed from natural products, showing particular efficacy against ovarian cancer. Camptothecin and analogues singly or combined with cisplatin show efficacy against solid tumors, breast, lung, and colorectal, which hitherto have been unaffected by most cancer chemotherapeutic agents [19].

The list of some plant derived drugs which can be served as a lead for the synthesis of new drugs is summarized in Table 1 .

\section{Challenges Faced By Natural Product Synthesis and Screening}

The total synthesis of complex natural products has long presented a big challenge to the synthetic chemist, and has led to considerable advances in synthetic methodology [20]. Enroute to the total synthesis of the natural product, it may be possible to identify a simpler precursor or analog containing the essential features of the molecule necessary for

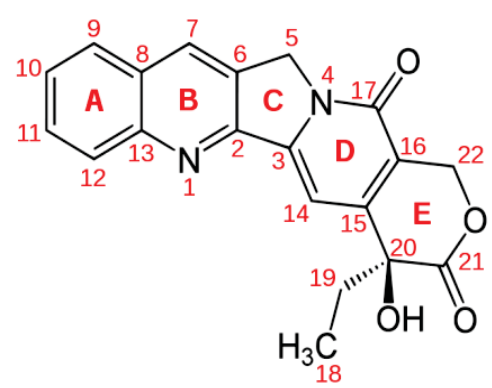

Figure 3: Chemical structure of Camptothecin.

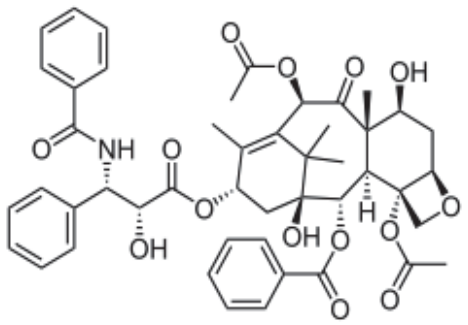

Figure 4: Chemical structure of Taxol (Paclitaxel). 
Citation: Pawar HA (2014) Natural Product as a Source of Lead to the Design of New Drugs. Nat Prod Chem Res 2: 156. doi:10.4172/23296836.1000156

\begin{tabular}{|l|l|}
\hline Plant Species & Plant Derived Drugs \\
\hline Adhatoda vasica Nees & Vasicine (peganine) \\
\hline Catharanthus roseus (L.) & Vinblastine (Navelbine) \\
\hline Papaver somniferum L & Papaverine \\
\hline Nicotiana tabacum L & Nicotine \\
\hline Cinchona ledgeriana & Quinidine \\
\hline Digitalis purpurea L & Digitoxin \\
\hline Ephedra sinica & Ephedrine \\
\hline Lobelia inflataL & Lobeline \\
\hline Physostigma venenosum & Physostigmine \\
\hline Rauvolfia serpentina & Reserpine \\
\hline Pilocarpus jaborandi & Pilocarpine \\
\hline Cephaelis ipecacuanha & Emetine \\
\hline
\end{tabular}

Table 1: List of plant derived drugs.

activity (the pharmacophore), and having similar or better activity. An important example is the synthesis of the marine-derived antitumor agent, halichondrin $\mathrm{B}$, which allowed the synthesis of a huge number of variants, mainly smaller molecules that maintained the biological activity, but were intrinsically more chemically stable, due to the substitution of a ketone for the ester linkage in the macrolide ring [21]. The advent of routine High Throughput Screening (HTS) has been one of the most important change to the drug discovery pre-process [2224]. Screening of one hundred thousand samples in a routine assay can now be completed in just over a week using 384-well formatting, a data handling system, and limited robotics. This screening time can be decreased further using higher density formats and advanced robotics $[25,26]$. Therefore, the number of compounds or extracts that can be screened for each drug target is generally not the rate-limiting step. More important considerations are the cost of the screen consumables, time required, and the people resources allocated to each screen. The cost of a screen can escalate quickly if an expensive screen format has to be used or the substrate or reagents are difficult to make, have a short half-life, and/or are expensive to purchase.

\section{Conclusion}

There is no doubt that plants are among the most perfect "natural laboratories" for the synthesis of various molecules ranging from simple skeleton to highly complex chemical structures. To continue to be competitive with other drug discovery methods, natural product research needs to continually improve the speed of the screening, isolation, and structure elucidation processes, as well addressing the suitability of screens for natural product extracts and dealing with issues involved with large-scale compound supply. Nature continues to be a main source of molecular diversity, which, through the pursuit of multidisciplinary, international, collaborative research, can result in the discovery of promising lead compounds, some of which may be developed into commercial drugs. The continuing threat to biodiversity through the destruction of terrestrial and marine ecosystems, however, lends urgency to the need to expand the collaborative exploration of these resources as a source of novel bioactive agents.

\section{References}

1. Farnsworth NR, Akerele O, Bingel AS, Soejarto DD, Guo Z (1985) Medicinal plants in therapy. Bull World Health Organ 63: 965-981.

2. Mitscher LA, Drake S, Gollapudi SR, Harris JA, Shankel DM (1986) Isolation and identification of higher plant agents active in antimutagenic assay systems: Glycyrrhiza glabra. Basic Life Sci 39: 153-165.

3. Williams DH, Stone MJ, Hauck PR, Rahman SK (1989) Why are secondary metabolites (natural products) biosynthesized? J Nat Prod 52: 1189-1208.

4. Woodbury AM, Wall ME, Willaman J (1961) Steroidal sapogenins. LV III. Steroidal sapogenins from the Joshua tree. Economic Botany 15: 79-86.
5. James F, Kerwin Jr. (1995) Burger's medicinal chemistry and drug discovery. 5th Edition. Volume 1: Principles and Practice. Manfred E. Wolff (ed.), WileyInterscience, New York. Drug Development Research 35: 116-117.

6. White NJ (2004) Antimalarial drug resistance. J Clin Invest 113: 1084-1092.

7. ter Kuile F, White NJ, Holloway P, Pasvol G, Krishna S (1993) Plasmodium falciparum: in vitro studies of the pharmacodynamic properties of drugs used for the treatment of severe malaria. Exp Parasitol 76: 85-95.

8. Dondorp A, Nosten F, Stepniewska K, Day N, White N; South East Asian Quinine Artesunate Malaria Trial (SEAQUAMAT) group (2005) Artesunate versus quinine for treatment of severe falciparum malaria: a randomised trial. Lancet 366: 717-725.

9. Woodrow CJ, Planche T, Krishna S (2006) Artesunate versus quinine for severe falciparum malaria. Lancet 367: 110-111.

10. Toovey $S$ (2006) Artesunate versus quinine for severe falciparum malaria Lancet 367: 111.

11. Bray PG, Ward SA, O'Neill PM (2005) Quinolines and artemisinin: chemistry, biology and history Curr Top Microbiol Immunol 295: 3-38.

12. Haynes RK (2001) Artemisinin and derivatives: the future for malaria treatment? Curr Opin Infect Dis 14: 719-726.

13. Haynes RK, Krishna S (2004) Artemisinins: activities and actions. Microbes Infect 6: 1339-1346.

14. Haynes RK, Ho WY, Chan HW, Fugmann B, Stetter J, et al. (2004) Highly antimalaria-active artemisinin derivatives: biological activity does not correlate with chemical reactivity. Angew Chem Int Ed Engl 43: 1381-1385.

15. Haynes RK (2005) Reply to comments on 'highly antimalaria-active artemisinin derivatives: biological activity does not correlate with chemical reactivity'. Angew Chem Int Ed Engl 44: 2064-2065.

16. Haynes RK, Fugmann B, Stetter J, Rieckmann K, Heilmann HD, et al. (2006) Artemisone--a highly active antimalarial drug of the artemisinin class. Angew Chem Int Ed Engl 45: 2082-2088.

17. Uhlemann AC, Cameron A, Eckstein-Ludwig U, Fischbarg J, Iserovich $P$, et al (2005) A single amino acid residue can determine the sensitivity of SERCAs to artemisinins. Nat Struct Mol Biol 12: 628-629.

18. Vennerstrom JL, Arbe-Barnes S, Brun R, Charman SA, Chiu FC, et al (2004) Identification of an antimalarial synthetic trioxolane drug development candidate. Nature 430: 900-904.

19. Wall ME, Wani MC (1995) Camptothecin and taxol: discovery to clinicthirteenth Bruce F. Cain Memorial Award Lecture. Cancer Res 55: 753-760.

20. Maureen RA (2003) Betting on Natural Products for Cures. Chem Eng News 81: 93-103.

21. Aicher TD, Buszek KR, Fang FG, Forsyth CJ, Jung SH, et al. (1992) Synthetic studies towards halichondrins: synthesis of the C.27-C.38 segment Tetrahedron Letters 33: 1549-1552.

22. Seethala R, Fernandes PB (2001) Handbook of Drug Screening; Drugs and the Pharmaceutical Sciences. New York: Marcel Dekker.

23. Carrano L, Donadio S (1999) In Combinatorial Chemistry and Technology: Principles, Methods, and Applications; Miertus S, Fassina G, Marcel Dekker New York, Chapter 10: 233-250.

24. Devlin JP (1997) High Throughput Screening: The Discovery of Bioactive Substances. New York: Marcel Dekker.

25. Vaschetto M, Weissbrod T, Bodle D, Güner O (2003) Enabling high-throughput discovery. Curr Opin Drug Discov Devel 6: 377-383.

26. Entzeroth M (2003) Emerging trends in high-throughput screening. Curr Opin Pharmacol 3: 522-529. 